\section{L'écopathologie appar-}

tient à un courant de recherches concernant l'agriculture et l'élevage dont les objets comportent les hommes, intervenant sur les choses non humaines. L'introduction des humains dans la construction de l'objet apporte du "désordre" dans le processus de recherche : par exemple la nécessité de sortir du laboratoire ou des élevages expérimentaux pour observer des élevages en conditions non contrôlées. Bien entendu, l'un des éléments les moins contrôlés du système, c'est l'éleveur lui-même, sa parole, ses comportements et ses humeurs.

Ainsi peuvent intervenir, dans le travail des chercheurs en sciences de la nature et de la vie, des difficultés qu'on peut dire de second degré : le recours aux sciences sociales. Je voudrais ici contribuer à transformer ces difficultés en pistes de recherches pour les auteurs. Le principal de mes observations porte sur l'usage du terme d'interaction et sur la façon dont les auteurs situent ce terme dans le champ des sciences sociales.

D'une façon générale dans l'article, le terme d'interaction est attribué - pour ce qui concerne son usage en sciences sociales - à un ensemble disciplinaire constitué des "sociologues et psycho-sociologues". On évoque des situations d'interaction « au sens où l'entendent sociologues et psycho-sociologues ". À partir de là surgissent plusieurs difficultés. Le terme d'interaction se situe dans plusieurs champs théoriques, de telle sorte qu'il n'est pas possible de parler d'un sens commun qu'il aurait dans les sciences sociales, et en particulier pour les sociologues et les psycho-sociologues. Les perspectives et arrièreplans des psycho-sociologues étudiant les interactions dans des petits groupes expérimentaux, pour en tirer des sortes de lois relatives aux réactions individuelles dans diverses situations de relations à d'autres, sont très loin d'être les mêmes que celles du sociologue N. Elias' analysant la façon dont Louis XIV exploite les systèmes d'interaction au sein de sa cour, que celle de E. Goffman' analysant les formes de Mise en scène de la vie quotidienne ou de A.V. Cicourel' montrant comment se construit la connaissance sociologique commune.

Les psycho-sociologues s'occupent de savoir comment les états individuels (attitudes, motivations, attrait/répulsion, représentations, etc.) s'investissent dans des situations. Chez les autres, sociologues et anthropologues, il s'agit avant tout de comprendre comment fonctionnent ou changent des systèmes de relations, et comment est constituée ou comment se construit et se transforme la connaissance qui règle ces fonctionnements, ou plus largement, toute connaissance.

Aujourd'hui, c'est généralement à ce champ que l'on se réfère lorsqu'on parle d'interaction en sciences sociales, champ associé aux approches "micro-sociologiques". Et l'on peut relever que certains courants psycho-sociologues voisinent de plus en plus avec ces sociologiest. C'était loin d'être le cas avec la psycho-sociologie des années trente aux ÉtatsUnis, et c'est loin d'être le cas avec sa descendance consacrée au "management des ressources humaines".

Je pense que la réduction opérée dans l'ensemble de l'article est dommageable pour le type de recherches que conduisent les auteurs, où figure cet intrus - pour les sciences de la nature et de la vie - qu'est l'individu conscient et connaissant. Les approches interactionnistes actuelles, avec des ouvrages pilotes tels que Studies in ethnomethodology de H. Garfinkel (1967 ; éditions Prentice Hall, Englewood Cliffs) et les livres de E. Goffman, ouvrent en effet à ces recherches deux domaines dans les voies indiquées par les auteurs dans leur conclusion. En premier lieu celui de la production de connaissance dans le dialogue (domaine auquel les auteurs consacrent un encadré), qui peut inviter à s'interroger sur la nature ou les caractéristiques de la connaissance produite dans les conditions particulières de l'enquête, et non plus seulement en termes dérivés de la notion de "biais". En deuxième lieu, celui de l'influence, sur les comportements du sujet, de l'environnement social de la situation, l'embeddedness (Polanyi), et sa position sociale, que les approches interactionnistes - sauf chez certains ethnométhodologues - introduisent expressément dans l'analyse ${ }^{6}$.

Par ailleurs, ces travaux incitent aussi à quelque prudence en ce qui concerne les possibilités et les conditions de la relation entre chercheur ou expert-conseil et éleveurs, relation qui comporte nécessairement, dans les perspectives des auteurs de l'article, une certaine dose de coopération. Ces travaux rappellent en particulier que, s'il est utile et légitime de reconnaitre la connaissance et les activités de production de connaissance aux sujets dans la vie quotidienne, face à la connaissance scientifique, on ne peut pas cependant parler de "symétrisation de la connaissance" dans la relation, parce que les positions des sujets sont dissymétriques, et parce que les formes de connaissance pour l'action et pour la recherche le sont aussi ${ }^{i}$. De même, sous ces éclairages, on ne peut pas retenir avec J. Piaget (encadré 3), et ce quelle que soit l'importance qu'on accorde d'autre part à son œuvre, que la coopération impliquerait " le renoncement à toute relation de domination n- et donc la possibilité de ce renoncement.

Ces travaux apportent donc, me semble-t-il, des possibilités de contributions à la recherche sur les "Rencontres du deuxième type" entre chercheurs et praticiens, à laquelle les auteurs de cet article apportent leur contribution. Malheureusement, ces travaux ne disent pas comment ils peuvent être utiles à de telles recherches, ils ne sont pas "prêts à l'emploi", à la différence, par exemple, des recherches directement liées à l'intervention ou à l'expertise. Cela invite à chercher et à expérimenter des moyens de "mise à disposition" réellement utiles, c'est-à-dire échappant aux simplifications, adaptations ou réductions au sens commun.
1. N. Elias, La société de cour, Paris, Calmann-Lèvy, 1974.
2. E. Goffman, La mise en scène de la vie quotidienne, Paris, Minuit, 1973.
3. A.V. Cicourel, La sociologie cognitive, Paris, PUF, 1979.
4. Cf. par exemple les contributions de D. Jodelet, W. Doise ou R. Harré dans $D$. Jodelet, Les représentations sociales, Paris, PUF, 1989.
5. Sur l'histoire et les orientations de ces travaux, $c$. le très bon ouvrage de
P. Demarez, Les sociologies industrielles aux États-Unis, Paris, A. Colin, 1986.
6. Angle d'approche theorise en particulier par A.V. Cicourel in K. Knorr-
Cetina et A.V. Cicourel, Advances in social theory and methodology. Toward an integration of micro and macro. sociologies, Boston Routledge and Kegan Paul, 1981. Voir aussi à ce propos E. Goffman, Asiles et Stigmates, Paris, Minuit, 1968 et 1975.
7. Thèses développées en particulier par
A. Schutz dans Le chercheur et le
quotidien, Paris, Klincksieck, Paris,
$1987)$; voir aussi Garfinkel déjà cité. 